\title{
To Study the Effect of the Generating Polynomial on the Quality of Nonlinear Components in Block Ciphers
}

\author{
Shahid Mahmood $(D),{ }^{1}$ Shabieh Farwa $\left(\mathbb{D},{ }^{2}\right.$ Muhammad Rafiq, ${ }^{2}$ \\ Syed Muhammad Jawwad Riaz, ${ }^{2}$ Tariq Shah, ${ }^{3}$ and Sajjad Shaukat Jamal ${ }^{3}$ \\ ${ }^{1}$ Department of Mechanical Engineering, Sarhad University of Science and Information Technology, Peshawar, Pakistan \\ ${ }^{2}$ Department of Mathematics, COMSATS Institute of Information Technology, Wah Cantt, Pakistan \\ ${ }^{3}$ Department of Mathematics, Quaid-i-Azam University, Islamabad, Pakistan \\ Correspondence should be addressed to Shabieh Farwa; drsfarwa@gmail.com
}

Received 1 November 2017; Revised 1 March 2018; Accepted 5 March 2018; Published 10 April 2018

Academic Editor: Amir Anees

Copyright (C) 2018 Shahid Mahmood et al. This is an open access article distributed under the Creative Commons Attribution License, which permits unrestricted use, distribution, and reproduction in any medium, provided the original work is properly cited.

\begin{abstract}
Substitution box (S-box), being the only nonlinear component, contributes to the confusion creating capability of a cryptosystem. Keeping in view the predominant role of $S$-box, many design algorithms to synthesize cryptographically stronger $S$-boxes have gained pivotal attention. A quick review of these algorithms shows that all these ideas mainly concentrate on the choice of bijective Boolean functions, with nonobservance to the irreducible polynomial that generates the Galois field. In this paper, we propose that the selection of irreducible polynomial has a deep influence on the highly desirable features of an $S$-box such as nonlinearity, strict avalanche, bit independence, linear approximation probability, and differential approximation probability. We underpin our claim by investigating a detailed model, which deploys the same algorithm but different polynomials and produces unusual changes in the results regarding the performance parameters of $S$-box.
\end{abstract}

\section{Introduction}

Electronic exchange of data has undoubtedly revolutionized the communication in recent years but, on the other hand, the secure transfer of confidential material over Internet has become the biggest challenge nowadays. It definitely demands seriously high level of security. The main problem is to avoid unauthorised access to the secret data. To achieve the desired level of security, many techniques such as cryptography, watermarking, and steganography have been the major focus of research for past few years [1-5]. In this paper, we deal with cryptography.

Cryptography is categorized into two types, symmetric key cryptography and asymmetric key cryptography. The symmetric key cryptography can be further split into two types: block ciphers and the stream ciphers. Advanced Encryption Standard is an example of block cipher that was officially adopted by the US government as the Federal Information Processing Standard (FIPS) in May 2002. AES algorithm [6] is based on four steps: round key addition, byte substitution, shift row, and mix column, but the most influential of all these is the byte substitution step. This step relies on a substitution box (S-box), which serves as the only nonlinear component in any substitution-permutation network (SPN).

It has been established that the substitution box (S-box) is a standout in different block ciphers and is a widely used mechanism in any substitution-permutation network as a source to produce nonlinearity [6]. It renders an absolutely complex, unforeseeable layout to requite various blocks of bits in output data. To extend high resistance against unexpected surveillance, $S$-box structure is required to fulfil certain standards. The indispensable involvement of $S$-box to induce complexity and nonlinearity motivates studying the properties and algorithms for safer and more reliable $S$-boxes. In this regard, many advanced structural developments are witnessed in literature. Khan et al. [7] proposed a technique for S-box construction based on chaotic Lorenz systems. Hussain et al. presented $S$-box algorithms using generalized Bakers map [8] and projective general linear group [9]. Algebraic, analytical, and chaotic approaches for $S$-box are 
studied in [10-13]. Özkaynak et al. [14] applied fractionalorder chaotic Chen system, to develop S-box. Tian and $\mathrm{Lu}$ [15] structured dynamic chaos-based $S$-box in conjunction with DNA sequence operation. Some other more efficient algorithms could be reviewed in $[6,16-20]$. In addition to this, applications of $S$-boxes in digital image encryption, steganography, and watermarking have become quite popular and influential in recent years $[4,11,13,21]$.

The study of innovation in design algorithms for $S$-boxes witnesses that the change of model and the selection of Boolean function contribute little to the performance indices of an $S$-box. We, in this paper, propose that the performance of an $S$-box is highly related to the background Galois field. The fact that finite fields of the same order are isomorphic is definitely of worth but the scrambling effect of a nonlinear Boolean function applied on two different fields of the same order might vary. Since in cryptography, an $S$-box is the salient component used to produce confusion in the data, it is worth studying that the confusion creating ability is associated with the choice of the irreducible polynomial used to form the background Galois field.

In [9], Hussain et al. presented an algorithm for generating $S$-box through the application of a linear fractional transformation on the Galois field $\operatorname{GF}\left(2^{8}\right)$, structured by the polynomial $X^{8}+X^{4}+X^{3}+X^{2}+1$. We in the proposed work show that the same algorithm used for a different polynomial exhibits highly improved values of nonlinearity, strict avalanche criterion (SAC), bit independent criterion (BIC), linear approximation probability (LAP), and differential approximation probability (DAP). By comparing the numerical results of these tests, we prove that different polynomials produce significantly different results. This observation leads to revising the existing models by choosing different background polynomials as it could be more influential in improvement of ideas rather changing the whole scheme.

We organize the contents of this paper as follows. In Section 2, we discuss the properties of the background Galois field $\operatorname{GF}\left(2^{8}\right)$. The detailed algorithm for the design of the $S$-box is presented in Section 3. Section 4 deals with the analyses of $S$-boxes against several common attacks and the comparison of respective results. We further compare the cryptographic standing of both of the newly synthesized $S$ boxes with the state-of-the-art AES S-box. Conclusion is presented in Section 5.

\section{Generating Polynomial and the Galois Field}

For any prime $p$, Galois field $\operatorname{GF}\left(p^{n}\right)$ is expressed as the factor ring $\mathbb{F}_{p}[X] /(\mu(x))$ where $\mu(x) \in \mathbb{F}_{p}[X]$ is an irreducible polynomial of degree $n$. For $\mathrm{GF}\left(2^{8}\right)$ we choose an irreducible polynomial of degree 8 that generates the maximal ideal of the principal ideal domain $\mathbb{F}_{2}[X]$. We know that the multiplicative group of the resultant field $\mathrm{GF}\left(2^{8}\right)$ is cyclic and hence each nonzero element of the field can be expressed as a power of the generator $\alpha=00000010$.

In order to support our claim regarding the effect of polynomial, we choose two irreducible primitive polynomials $\mu_{1}$ and $\mu_{2}$ of degree 8 , to construct Galois fields $\mathbb{F}_{1}$ and $\mathbb{F}_{2}$, respectively, where $\mu_{1}=X^{8}+X^{6}+X^{5}+X^{4}+1$ and $\mu_{2}=X^{8}+X^{4}+X^{3}+X^{2}+1$, as used in [9]. We may choose other polynomials as well to compare our calculations but the selected pair beautifully serves for the purpose. Let $G_{i}$ represents the multiplicative group of the Galois field, $\mathbb{F}_{i}$. The exponential form of elements of the multiplicative group $G_{1}$, along with their inverses, is represented in Table 1; however the elements of $G_{2}$ are presented in Table 2 of [9]. In the next section, we use these calculations to develop the corresponding $S$-boxes.

\section{Algorithm for $S$-Box}

An $n \times n S$-box is defined by a vector Boolean function $\mathcal{S}_{n}$ : $\mathrm{GF}\left(2^{n}\right) \rightarrow \mathrm{GF}\left(2^{n}\right)$, defined as

$$
\delta_{n}(v)=\left(s_{1}(v), s_{2}(v), \ldots, s_{n}(v)\right),
$$

where $v=\left(v_{1}, v_{2}, \ldots, v_{2^{n}}\right) \in \mathrm{GF}\left(2^{n}\right)$ and each of $s_{i}$ 's is regarded as a component Boolean function.

For a field $\mathbb{F}$, the general linear group $\mathrm{GL}(n, \mathbb{F})$ is a group formed by all $n \times n$ invertible matrices. A projective general linear group of degree $n$ over a field $\mathbb{F}$ is defined to be the quotient group of $\operatorname{GL}(n, \mathbb{F})$ by its center. For this paper, we form the $8 \times 8 S$-box by considering the action of the Galois field $\mathrm{GF}\left(2^{8}\right)$ on the projective linear group $\operatorname{PGL}\left(2, \operatorname{GF}\left(2^{8}\right)\right)$; that is, we take a function $\sigma: \operatorname{PGL}\left(2, \operatorname{GF}\left(2^{8}\right)\right) \times \operatorname{GF}\left(2^{8}\right) \rightarrow$ $\mathrm{GF}\left(2^{8}\right)$ defined as follows:

$$
\sigma(v)=\frac{\alpha v+\beta}{\gamma v+\delta} .
$$

In (2), $\sigma$ is known as a linear fractional transformation (LFT) with $\alpha, \beta, \gamma$ and $\delta \in \mathrm{GF}\left(2^{8}\right)$ satisfying the nondegeneracy condition $\alpha \delta-\beta \gamma \neq 0$. The ease of implementation, lesser computational labour, and high algebraic complexity of an LFT are the prime features that give incentive to employ this map for byte substitution. We may choose any values for LFT parameters that satisfy the aforementioned condition but, for the presented calculations, we, in particular, choose the same values as in [9], so that a comparison could be set easily. We consider $\alpha=35, \beta=15, \gamma=9$ and $\delta=5$. The images of the map $\sigma$, when applied on $\mathbb{F}_{1}$ and $\mathbb{F}_{2}$, produce our $S$-boxes $S_{1}$ and $S_{2}$, respectively, as shown in Tables 2 and 3 .

\section{Performance Analysis of $S$-Boxes}

The cryptographic strength if the $S$-boxes, generated in the foregoing section, are examined through the most widely used analysis techniques such as nonlinearity, bit independence, strict avalanche, and linear and differential approximation probabilities. In the following subsections we present all these performance indices one by one and compare the performance of $S_{1}$ and $S_{2}$ with one another, as well as, with the ever-prevailing algorithm AES.

4.1. Nonlinearity. Nonlinearity analysis measures the distance of the reference function from all of the affine functions. 
TABLE 1: Exponential representation and the multiplicative inverses of elements of $G_{1}$.

\begin{tabular}{|c|c|c|}
\hline$x \in G_{1}$ & $\alpha^{n}$ & $x^{-1}$ \\
\hline 1 & $\alpha^{255}$ & 1 \\
\hline 2 & $\alpha^{1}$ & 184 \\
\hline 3 & $\alpha^{231}$ & 208 \\
\hline 4 & $\alpha^{2}$ & 92 \\
\hline 5 & $\alpha^{207}$ & 159 \\
\hline 6 & $\alpha^{232}$ & 104 \\
\hline 7 & $\alpha^{59}$ & 134 \\
\hline 8 & $\alpha^{3}$ & 46 \\
\hline 9 & $\alpha^{35}$ & 173 \\
\hline 10 & $\alpha^{208}$ & 247 \\
\hline 11 & $\alpha^{154}$ & 139 \\
\hline 12 & $\alpha^{233}$ & 52 \\
\hline 13 & $\alpha^{20}$ & 48 \\
\hline 14 & $\alpha^{60}$ & 67 \\
\hline 15 & $\alpha^{183}$ & 117 \\
\hline 16 & $\alpha^{4}$ & 23 \\
\hline 17 & $\alpha^{159}$ & 252 \\
\hline 18 & $\alpha^{36}$ & 238 \\
\hline 19 & $\alpha^{66}$ & 83 \\
\hline 20 & $\alpha^{209}$ & 195 \\
\hline 21 & $\alpha^{118}$ & 204 \\
\hline 22 & $\alpha^{155}$ & 253 \\
\hline 23 & $\alpha^{251}$ & 16 \\
\hline 24 & $\alpha^{234}$ & 26 \\
\hline 25 & $\alpha^{245}$ & 181 \\
\hline 26 & $\alpha^{21}$ & 24 \\
\hline 27 & $\alpha^{11}$ & 180 \\
\hline 28 & $\alpha^{61}$ & 153 \\
\hline 29 & $\alpha^{130}$ & 121 \\
\hline 30 & $\alpha^{184}$ & 130 \\
\hline 31 & $\alpha^{146}$ & 73 \\
\hline 32 & $\alpha^{5}$ & 179 \\
\hline 33 & $\alpha^{122}$ & 232 \\
\hline 34 & $\alpha^{160}$ & 126 \\
\hline 35 & $\alpha^{79}$ & 141 \\
\hline 36 & $\alpha^{37}$ & 119 \\
\hline 37 & $\alpha^{113}$ & 220 \\
\hline 38 & $\alpha^{67}$ & 145 \\
\hline 39 & $\alpha^{106}$ & 248 \\
\hline 40 & $\alpha^{210}$ & 217 \\
\hline 41 & $\alpha^{224}$ & 175 \\
\hline 42 & $\alpha^{119}$ & 102 \\
\hline 43 & $\alpha^{221}$ & 188 \\
\hline 44 & $\alpha^{156}$ & 198 \\
\hline 45 & $\alpha^{242}$ & 108 \\
\hline 46 & $\alpha^{252}$ & 8 \\
\hline 47 & $\alpha^{32}$ & 172 \\
\hline 48 & $\alpha^{235}$ & 13 \\
\hline 49 & $\alpha^{213}$ & 53 \\
\hline 50 & $\alpha^{246}$ & 226 \\
\hline 51 & $\alpha^{135}$ & 84 \\
\hline 52 & $\alpha^{22}$ & 12 \\
\hline 53 & $\alpha^{42}$ & 49 \\
\hline 54 & $\alpha^{12}$ & 90 \\
\hline
\end{tabular}

TABLE 1: Continued.

\begin{tabular}{lcc}
\hline$x \in G_{1}$ & $\alpha^{n}$ & $x^{-1}$ \\
\hline 55 & $\alpha^{140}$ & 148 \\
56 & $\alpha^{62}$ & 244 \\
57 & $\alpha^{227}$ & 223 \\
58 & $\alpha^{131}$ & 132 \\
59 & $\alpha^{75}$ & 203 \\
60 & $\alpha^{185}$ & 65 \\
61 & $\alpha^{191}$ & 224
\end{tabular}

62

63

64

65

66

67

68

69

70

71

72

73

74

75

76

77

78

79

80

81

82

83

84

85

86

87

88

89

90

91

92

93

94

95

96

96
97

98

99

100

101

102

103

104

105

106

107 $x^{-1}$
48
23
32
65
224

156

68

225

60

116

14

63

157

254

143

131

31

110

154

240

150

124

186

212

196

239

19

51

227

94

193

99

163

54

149

4

158

86

192

190

235

162

88

113

123

42

189 
TABle 1: Continued.

\begin{tabular}{|c|c|c|}
\hline$x \in G_{1}$ & $\alpha^{n}$ & $x^{-1}$ \\
\hline 108 & $\alpha^{13}$ & 45 \\
\hline 109 & $\alpha^{169}$ & 199 \\
\hline 110 & $\alpha^{141}$ & 74 \\
\hline 111 & $\alpha^{89}$ & 155 \\
\hline 112 & $\alpha^{63}$ & 122 \\
\hline 113 & $\alpha^{8}$ & 100 \\
\hline 114 & $\alpha^{228}$ & 215 \\
\hline 115 & $\alpha^{151}$ & 237 \\
\hline 116 & $\alpha^{132}$ & 66 \\
\hline 117 & $\alpha^{72}$ & 15 \\
\hline 118 & $\alpha^{76}$ & 221 \\
\hline 119 & $\alpha^{218}$ & 36 \\
\hline 120 & $\alpha^{186}$ & 152 \\
\hline 121 & $\alpha^{125}$ & 29 \\
\hline 122 & $\alpha^{192}$ & 112 \\
\hline 123 & $\alpha^{200}$ & 101 \\
\hline 124 & $\alpha^{148}$ & 78 \\
\hline 125 & $\alpha^{197}$ & 187 \\
\hline 126 & $\alpha^{95}$ & 34 \\
\hline 127 & $\alpha^{174}$ & 140 \\
\hline 128 & $\alpha^{7}$ & 200 \\
\hline 129 & $\alpha^{150}$ & 171 \\
\hline 130 & $\alpha^{71}$ & 30 \\
\hline 131 & $\alpha^{217}$ & 72 \\
\hline 132 & $\alpha^{124}$ & 58 \\
\hline 133 & $\alpha^{199}$ & 202 \\
\hline 134 & $\alpha^{196}$ & 7 \\
\hline 135 & $\alpha^{173}$ & 105 \\
\hline 136 & $\alpha^{162}$ & 167 \\
\hline 137 & $\alpha^{97}$ & 176 \\
\hline 138 & $\alpha^{54}$ & 246 \\
\hline 139 & $\alpha^{101}$ & 11 \\
\hline 140 & $\alpha^{81}$ & 127 \\
\hline 141 & $\alpha^{176}$ & 35 \\
\hline 142 & $\alpha^{168}$ & 255 \\
\hline 143 & $\alpha^{88}$ & 71 \\
\hline 144 & $\alpha^{39}$ & 249 \\
\hline 145 & $\alpha^{188}$ & 38 \\
\hline 146 & $\alpha^{110}$ & 183 \\
\hline 147 & $\alpha^{239}$ & 243 \\
\hline 148 & $\alpha^{115}$ & 55 \\
\hline 149 & $\alpha^{127}$ & 91 \\
\hline 150 & $\alpha^{204}$ & 77 \\
\hline 151 & $\alpha^{17}$ & 241 \\
\hline 152 & $\alpha^{69}$ & 120 \\
\hline 153 & $\alpha^{194}$ & 28 \\
\hline 154 & $\alpha^{52}$ & 75 \\
\hline 155 & $\alpha^{166}$ & 111 \\
\hline 156 & $\alpha^{108}$ & 62 \\
\hline 157 & $\alpha^{202}$ & 69 \\
\hline 158 & $\alpha^{50}$ & 93 \\
\hline 159 & $\alpha^{48}$ & 5 \\
\hline 160 & $\alpha^{212}$ & 106 \\
\hline 161 & $\alpha^{134}$ & 168 \\
\hline 162 & $\alpha^{41}$ & 98 \\
\hline
\end{tabular}

TABLE 1: Continued.

\begin{tabular}{|c|c|c|}
\hline$x \in G_{1}$ & $\alpha^{n}$ & $x^{-1}$ \\
\hline 163 & $\alpha^{139}$ & 89 \\
\hline 164 & $\alpha^{226}$ & 207 \\
\hline 165 & $\alpha^{74}$ & 231 \\
\hline 166 & $\alpha^{190}$ & 177 \\
\hline 167 & $\alpha^{93}$ & 136 \\
\hline 168 & $\alpha^{121}$ & 161 \\
\hline 169 & $\alpha^{78}$ & 107 \\
\hline 170 & $\alpha^{112}$ & 201 \\
\hline 171 & $\alpha^{105}$ & 129 \\
\hline 172 & $\alpha^{223}$ & 47 \\
\hline 173 & $\alpha^{220}$ & 9 \\
\hline 174 & $\alpha^{241}$ & 216 \\
\hline 175 & $\alpha^{31}$ & 41 \\
\hline 176 & $\alpha^{158}$ & 137 \\
\hline 177 & $\alpha^{65}$ & 166 \\
\hline 178 & $\alpha^{117}$ & 233 \\
\hline 179 & $\alpha^{250}$ & 32 \\
\hline 180 & $\alpha^{244}$ & 27 \\
\hline 181 & $\alpha^{10}$ & 25 \\
\hline 182 & $\alpha^{129}$ & 242 \\
\hline 183 & $\alpha^{145}$ & 146 \\
\hline 184 & $\alpha^{254}$ & 2 \\
\hline 185 & $\alpha^{230}$ & 209 \\
\hline 186 & $\alpha^{206}$ & 79 \\
\hline 187 & $\alpha^{58}$ & 125 \\
\hline 188 & $\alpha^{34}$ & 43 \\
\hline 189 & $\alpha^{153}$ & 103 \\
\hline 190 & $\alpha^{19}$ & 96 \\
\hline 191 & $\alpha^{182}$ & 234 \\
\hline 192 & $\alpha^{237}$ & 95 \\
\hline 193 & $\alpha^{15}$ & 87 \\
\hline 194 & $\alpha^{164}$ & 205 \\
\hline 195 & $\alpha^{46}$ & 20 \\
\hline 196 & $\alpha^{215}$ & 81 \\
\hline 197 & $\alpha^{171}$ & 213 \\
\hline 198 & $\alpha^{99}$ & 44 \\
\hline 199 & $\alpha^{86}$ & 109 \\
\hline 200 & $\alpha^{248}$ & 128 \\
\hline 201 & $\alpha^{143}$ & 170 \\
\hline 202 & $\alpha^{56}$ & 133 \\
\hline 203 & $\alpha^{180}$ & 59 \\
\hline 204 & $\alpha^{137}$ & 21 \\
\hline 205 & $\alpha^{91}$ & 194 \\
\hline 206 & $\alpha^{103}$ & 230 \\
\hline 207 & $\alpha^{29}$ & 164 \\
\hline 208 & $\alpha^{24}$ & 3 \\
\hline 209 & $\alpha^{25}$ & 185 \\
\hline 210 & $\alpha^{83}$ & 251 \\
\hline 211 & $\alpha^{26}$ & 228 \\
\hline 212 & $\alpha^{44}$ & 80 \\
\hline 213 & $\alpha^{84}$ & 197 \\
\hline 214 & $\alpha^{178}$ & 236 \\
\hline 215 & $\alpha^{27}$ & 114 \\
\hline 216 & $\alpha^{14}$ & 174 \\
\hline 217 & $\alpha^{45}$ & 40 \\
\hline
\end{tabular}


TABle 1: Continued.

\begin{tabular}{|c|c|c|}
\hline$x \in G_{1}$ & $\alpha^{n}$ & $x^{-1}$ \\
\hline 218 & $\alpha^{170}$ & 219 \\
\hline 219 & $\alpha^{85}$ & 218 \\
\hline 220 & $\alpha^{142}$ & 37 \\
\hline 221 & $\alpha^{179}$ & 118 \\
\hline 222 & $\alpha^{90}$ & 245 \\
\hline 223 & $\alpha^{28}$ & 57 \\
\hline 224 & $\alpha^{64}$ & 61 \\
\hline 225 & $\alpha^{249}$ & 64 \\
\hline 226 & $\alpha^{9}$ & 50 \\
\hline 227 & $\alpha^{144}$ & 85 \\
\hline 228 & $\alpha^{229}$ & 211 \\
\hline 229 & $\alpha^{57}$ & 250 \\
\hline 230 & $\alpha^{152}$ & 206 \\
\hline 231 & $\alpha^{181}$ & 165 \\
\hline 232 & $\alpha^{133}$ & 33 \\
\hline 233 & $\alpha^{138}$ & 178 \\
\hline 234 & $\alpha^{73}$ & 191 \\
\hline 235 & $\alpha^{92}$ & 97 \\
\hline 236 & $\alpha^{77}$ & 214 \\
\hline 237 & $\alpha^{104}$ & 115 \\
\hline 238 & $\alpha^{219}$ & 18 \\
\hline 239 & $\alpha^{30}$ & 82 \\
\hline 240 & $\alpha^{187}$ & 76 \\
\hline 241 & $\alpha^{238}$ & 151 \\
\hline 242 & $\alpha^{126}$ & 182 \\
\hline 243 & $\alpha^{16}$ & 147 \\
\hline 244 & $\alpha^{193}$ & 56 \\
\hline 245 & $\alpha^{165}$ & 222 \\
\hline 246 & $\alpha^{201}$ & 138 \\
\hline 247 & $\alpha^{47}$ & 10 \\
\hline 248 & $\alpha^{149}$ & 39 \\
\hline 249 & $\alpha^{216}$ & 144 \\
\hline 250 & $\alpha^{198}$ & 229 \\
\hline 251 & $\alpha^{172}$ & 210 \\
\hline 252 & $\alpha^{96}$ & 17 \\
\hline 253 & $\alpha^{100}$ & 22 \\
\hline 254 & $\alpha^{175}$ & 70 \\
\hline 255 & $\alpha^{87}$ & 142 \\
\hline
\end{tabular}

Nonlinearity criterion outlines the total number of bits that must be altered in the truth table of a Boolean function to get close to the nearby affine function [22].

Table 4 shows that, for $S_{1}$, the average nonlinearity measure is 112., which is the highest figure attained by the AES $S$-box. Figure 1 shows the comparison which clearly depicts outstanding performance of $S_{1}$ as compared to $\mathrm{S}_{2}$.

4.2. Linear Approximation Probability. The measure of unevenness of an event is determined by linear approximation probability. This analysis is used to evaluate the maximum imbalance of the outcome. Mathematically, the linear approximation probability for a given $S$-box is defined as follows:

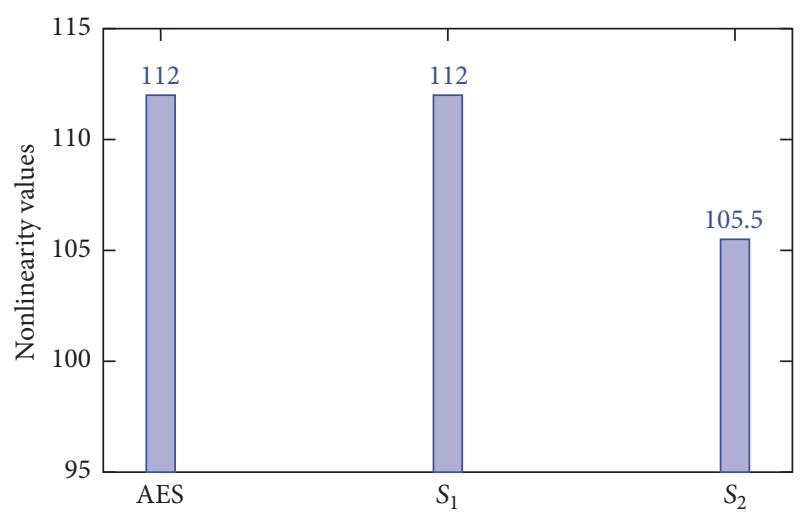

FIGURE 1: Nonlinearity of different $S$-boxes.

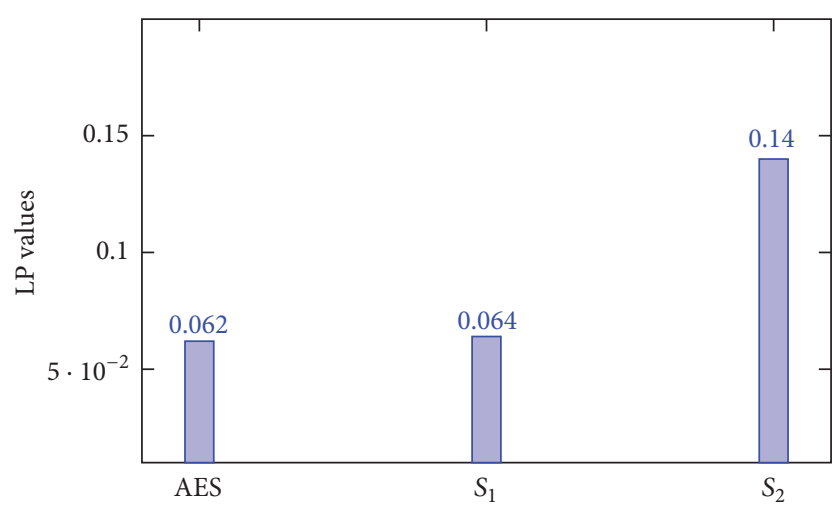

FIGURE 2: LP of different $S$-boxes.

$$
\mathrm{LP}=\max _{\Gamma_{x}, \Gamma_{y} \neq 0}\left|\frac{\#\left\{x \mid x \cdot \Gamma_{x}=S(x) \cdot \Gamma_{y}\right\}}{2^{n}}-\frac{1}{2}\right|,
$$

where $X$ represents the set of all possible inputs and $\Gamma_{x}$ and $\Gamma_{y}$ are the input and output masks, respectively. Numerical results presented in Table 5 and compared in Figure 2 show that the linear approximation probability of $S_{1}$ is much better than $S_{2}$.

4.3. Differential Approximation Probability. For further analysis, we use the differential approximation probability, which determines the differential uniformity demonstrated by an $S$-box. The mathematical expression for DP is given by the following:

$$
\mathrm{DP}=\left[\frac{\#\{x \in X \mid S(x) \oplus S(x \oplus \Delta x)=\Delta y\}}{2^{n}}\right] .
$$

In the above-mentioned expression input and output differentials are represented by $\Delta x$ and $\Delta y$, respectively. The smaller the differential uniformity, the stronger the $S$-box. It is evident from Table 5 and Figure 3 that in terms of the differential approximation probability $S_{1}$ is much stronger than $S_{2}$.

4.4. Strict Avalanche Criterion. This criterion examines the changes in the output bits caused as a result of single input 
TABLE 2: $S$-box $S_{1}$.

\begin{tabular}{|c|c|c|c|c|c|c|c|c|c|c|c|c|c|c|c|}
\hline 3 & 214 & 37 & 74 & 126 & 4 & 18 & 26 & 219 & 62 & 45 & 226 & 50 & 136 & 104 & 148 \\
\hline 154 & 38 & 200 & 199 & 185 & 228 & 170 & 245 & 177 & 114 & 137 & 231 & 139 & 35 & 8 & 134 \\
\hline 158 & 27 & 223 & 232 & 17 & 157 & 217 & 49 & 83 & 141 & 171 & 42 & 47 & 206 & 64 & 194 \\
\hline 106 & 29 & 30 & 110 & 14 & 40 & 72 & 236 & 105 & 221 & 202 & 87 & 241 & 41 & 11 & 71 \\
\hline 186 & 28 & 253 & 175 & 67 & 31 & 23 & 33 & 66 & 189 & 117 & 118 & 94 & 149 & 135 & 252 \\
\hline 235 & 43 & 124 & 125 & 90 & 229 & 204 & 215 & 218 & 100 & 101 & 249 & 243 & 54 & 173 & 166 \\
\hline 138 & 234 & 244 & 201 & 167 & 44 & 250 & 25 & 16 & 187 & 207 & 246 & 107 & 103 & 161 & 1 \\
\hline 183 & 99 & 179 & 240 & 129 & 123 & 188 & 193 & 20 & 143 & 155 & 174 & 7 & 220 & 213 & 239 \\
\hline 108 & 84 & 113 & 184 & 5 & 57 & 208 & 153 & 75 & 112 & 223 & 178 & 180 & 150 & 65 & 24 \\
\hline 224 & 248 & 102 & 89 & 70 & 111 & 59 & 172 & 95 & 131 & 198 & 163 & 93 & 164 & 55 & 209 \\
\hline 86 & 132 & 6 & 225 & 51 & 79 & 53 & 34 & 97 & 48 & 197 & 142 & 182 & 210 & 91 & 247 \\
\hline 195 & 15 & 10 & 144 & 85 & 63 & 168 & 238 & 196 & 162 & 98 & 32 & 251 & 254 & 203 & 156 \\
\hline 80 & 152 & 237 & 24 & 127 & 78 & 165 & 12 & 52 & 222 & 122 & 58 & 211 & 36 & 140 & 191 \\
\hline 216 & 146 & 109 & 96 & 147 & 73 & 116 & 190 & 128 & 68 & 56 & 77 & 115 & 160 & 19 & 92 \\
\hline 69 & 2 & 192 & 121 & 145 & 21 & 76 & 61 & 60 & 181 & 133 & 151 & 159 & 0 & 88 & 205 \\
\hline 13 & 230 & 22 & 82 & 39 & 119 & 9 & 255 & 120 & 46 & 81 & 212 & 227 & 130 & 176 & 169 \\
\hline
\end{tabular}

TABle 3: $S$-box $S_{2}$.

\begin{tabular}{|c|c|c|c|c|c|c|c|c|c|c|c|c|c|c|c|}
\hline 198 & 214 & 241 & 163 & 130 & 165 & 217 & 127 & 179 & 123 & 111 & 197 & 43 & 141 & 237 & 3 \\
\hline 168 & 201 & 17 & 121 & 142 & 101 & 232 & 174 & 11 & 249 & 16 & 156 & 10 & 50 & 183 & 65 \\
\hline 72 & 184 & 200 & 132 & 58 & 47 & 27 & 159 & 231 & 189 & 8 & 18 & 206 & 194 & 177 & 31 \\
\hline 193 & 92 & 122 & 192 & 85 & 137 & 243 & 49 & 178 & 170 & 36 & 135 & 230 & 95 & 100 & 128 \\
\hline 13 & 109 & 227 & 0 & 224 & 144 & 208 & 78 & 173 & 32 & 139 & 234 & 107 & 82 & 172 & 81 \\
\hline 51 & 233 & 12 & 154 & 94 & 161 & 244 & 55 & 7 & 34 & 251 & 225 & 153 & 93 & 254 & 138 \\
\hline 102 & 240 & 115 & 242 & 110 & 134 & 124 & 79 & 157 & 160 & 90 & 238 & 73 & 53 & 169 & 250 \\
\hline 136 & 118 & 112 & 48 & 40 & 114 & 22 & 246 & 46 & 131 & 23 & 69 & 52 & 235 & 248 & 2 \\
\hline 116 & 91 & 117 & 26 & 166 & 25 & 219 & 59 & 54 & 229 & 120 & 245 & 89 & 185 & 99 & 226 \\
\hline 105 & 45 & 60 & 199 & 164 & 191 & 228 & 202 & 37 & 104 & 143 & 209 & 220 & 147 & 44 & 186 \\
\hline 145 & 125 & 203 & 29 & 38 & 41 & 215 & 108 & 64 & 88 & 119 & 74 & 213 & 96 & 211 & 83 \\
\hline 218 & 146 & 196 & 205 & 67 & 152 & 129 & 175 & 84 & 158 & 207 & 176 & 80 & 62 & 150 & 86 \\
\hline 57 & 155 & 195 & 216 & 75 & 19 & 1 & 87 & 33 & 68 & 71 & 236 & 239 & 255 & 35 & 212 \\
\hline 148 & 188 & 133 & 15 & 204 & 187 & 42 & 182 & 97 & 56 & 24 & 221 & 252 & 30 & 77 & 181 \\
\hline 4 & 247 & 167 & 21 & 9 & 222 & 180 & 190 & 151 & 140 & 39 & 171 & 14 & 126 & 66 & 253 \\
\hline 103 & 223 & 70 & 98 & 28 & 20 & 63 & 162 & 61 & 113 & 149 & 210 & 106 & 5 & 6 & 76 \\
\hline
\end{tabular}

bit change. This is one of the most desirable features of any cryptographic design that when we change a single input bit, changes must occur in half of the output bits. In other words an $S$-box, $\mathcal{S}_{n}: \mathbb{F}_{2}^{n} \rightarrow \mathbb{F}_{2}^{n}$ is said to satisfy SAC if, for a change in an input bit, the probability of change in the output bit is $1 / 2$. The results are shown in Table 5 and Figure 4.

4.5. Bit Independence Criterion. The independent behavior of the pair of variables and the variations of input bits are considered as important factors of bit independence criterion. In bit independence criterion, input bits are transformed exclusively, and then output results are scrutinized for their independency [23]. Bit independence has great worth in cryptographic structures. The goal of reaching the maximum complexity and perplexity in a system can be achieved through this property of increasing independence between the bits. In cryptographic systems, the increased independence between bits is an essential requirement as it makes harder to understand and forecast the design of the system.

The numerical results of BIC when applied to the proposed $S$-box are given in Table 5 and are compared in Figure 5. It can be observed that according to these results our $S$-box $S_{1}$ is pretty similar to the AES $S$-box and is much better than $S_{2}$.

One can observe that overall performance of $S_{1}$ is much better than that of $S_{2}$. The performance parameters for $S_{1}$ seem to be pretty close to that of AES $S$-box. The algorithm used for both $S_{1}$ and $S_{2}$ is the same but the primitive polynomial selected to generate the Galois field is different, which really contributes to the outputs.

\section{Conclusion}

The kernel of the presented work lies in the fact that the choice of the background Galois field and its generating primitive 
TABLE 4: Performance Indices for new $S$-box.

\begin{tabular}{lccccc}
\hline Analysis & Max. & Min. & Average & Square deviation & DP \\
\hline Nonlinearity & 113 & 111 & $\mathbf{1 1 2}$ & & LP \\
SAC & 0.546875 & 0.429688 & $\mathbf{0 . 4 9 8 2 9 1}$ & 0.0157537 & 0.6227 \\
BIC & & 111 & $\mathbf{1 1 1 . 7 5 1}$ & & $\mathbf{0 . 0 1 5 6 2 5}$ \\
DP & & & & & $\mathbf{0 . 0 6 4 0 6 3}$
\end{tabular}

TABLE 5: Comparison of performance indices for different $S$-boxes.

\begin{tabular}{lccccc}
\hline$S$-box & Nonlinearity & SAC & BIC & DP & LP \\
\hline AES & 112 & 0.5058 & 112.0 & 0.0156 & 0.062 \\
$S_{1}$ & $\mathbf{1 1 2}$ & $\mathbf{0 . 4 9 8 2 9 1}$ & $\mathbf{1 1 1 . 7 5 1}$ & $\mathbf{0 . 0 1 5 6 2 5}$ & $\mathbf{0 . 0 6 4 0 6 3}$ \\
$S_{2}$ & $\mathbf{1 0 5 . 5}$ & $\mathbf{0 . 5 0 7}$ & $\mathbf{1 0 6}$ & $\mathbf{0 . 0 2 4 2}$ & $\mathbf{0 . 1 4 0}$ \\
\hline
\end{tabular}

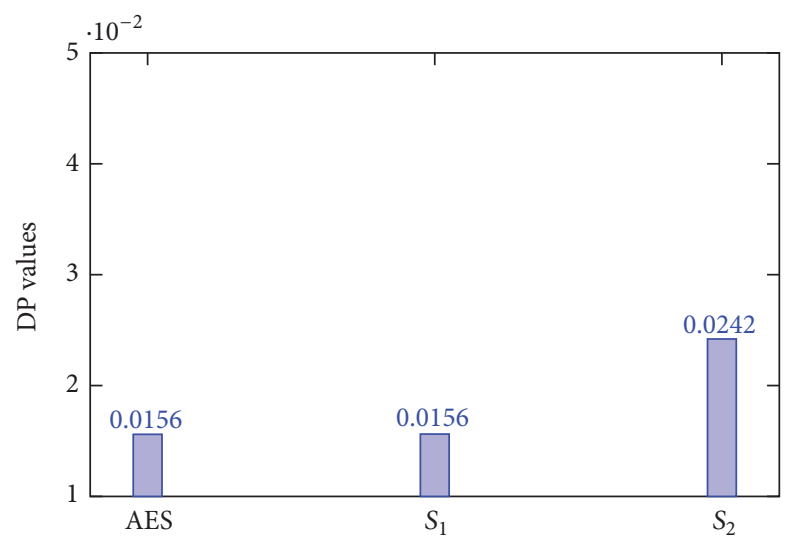

FIgURE 3: DP of different $S$-boxes.

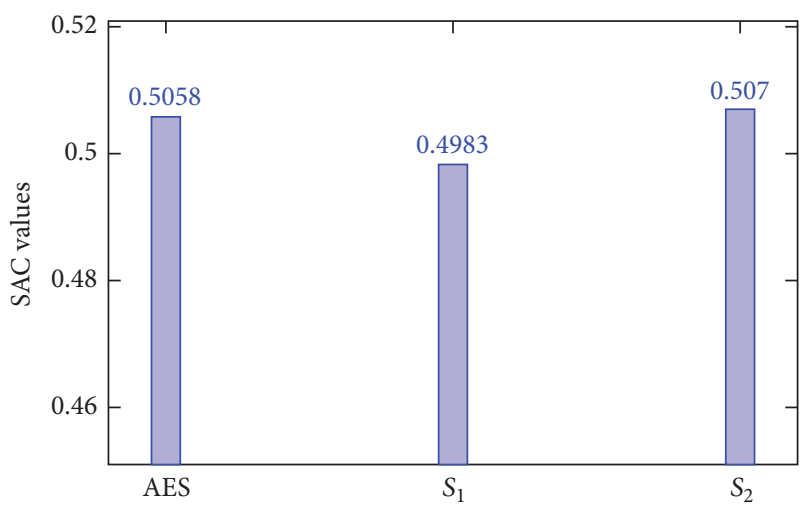

Figure 4: SAC of different $S$-boxes.

polynomial matters to the function and performance of the substitution boxes. This fact leads to the fascinating idea that, rather than the development of new algorithms, the improvement of the existing algorithms is worth studying as its least laborious but most effective. We propose, on the basis of the example discussed, that the effect of the choice of generating polynomial may lead to an intensive research in future to modify the design models of $S$-boxes. It will definitely affect the applications of $S$-boxes in other branches of the digital

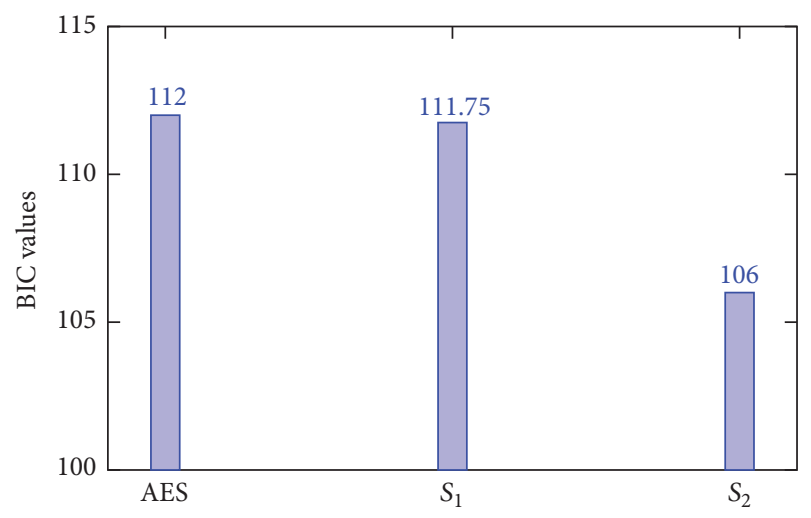

FIgURE 5: BIC of different $S$-boxes.

communication, such as steganography, watermarking, and image encryption.

\section{Conflicts of Interest}

The authors declare that there are no conflicts of interest regarding the publication of this paper.

\section{Acknowledgments}

The authors are grateful to the Sarhad University of Science and Information Technology (Pakistan), for providing partial funding for this research work.

\section{References}

[1] C. E. Shannon, "Communication theory of secrecy systems," Bell Labs Technical Journal, vol. 28, pp. 656-715, 1949.

[2] H. Feistel, "Cryptography and computer privacy," Scientific American, vol. 228, no. 5, pp. 15-23, 1973.

[3] A. Cheddad, J. Condell, K. Curran, and P. Mc Kevitt, "Digital image steganography: survey and analysis of current methods," Signal Processing, vol. 90, no. 3, pp. 727-752, 2010.

[4] S. S. Jamal, T. Shah, S. Farwa, and M. U. Khan, "A new technique of frequency domain watermarking based on a local ring," Wireless Networks.

[5] S. S. Jamal, T. Shah, and I. Hussain, "An efficient scheme for digital watermarking using chaotic map," Nonlinear Dynamics, vol. 73, no. 3, pp. 1469-1474, 2013.

[6] J. Daemen and V. Rijmen, The Design of Rijndael: AES-The Advanced Encryption Standard, Springer, Berlin, Germany, 2002. 
[7] M. Khan, T. Shah, H. Mahmood, M. A. Gondal, and I. Hussain, "A novel technique for the construction of strong S-boxes based on chaotic Lorenz systems," Nonlinear Dynamics, vol. 70, no. 3, pp. 2303-2311, 2012.

[8] I. Hussain, T. Shah, M. A. Gondal, and H. Mahmood, "Efficient method for designing chaotic S-boxes based on generalized Baker's map and TDERC chaotic sequence," Nonlinear Dynamics, vol. 74, no. 1-2, pp. 271-275, 2013.

[9] I. Hussain, T. Shah, H. Mahmood, and M. A. Gondal, "A projective general linear group based algorithm for the construction of substitution box for block ciphers," Neural Computing and Applications, vol. 22, no. 6, pp. 1085-1093, 2013.

[10] S. Farwa, N. Muhammad, T. Shah, and S. Ahmad, "A Novel Image Encryption Based on Algebraic S-box and Arnold Transform," 3D Research, vol. 8, no. 3, article no. 26, 2017.

[11] S. Farwa, T. Shah, and L. Idrees, "A highly nonlinear S-box based on a fractional linear transformation," SpringerPlus, vol. 5, no. 1, article no. 1658, 2016.

[12] S. V. Radhakrishnan and S. Subramanian, "An analytical approach to s-box generation," Computers and Electrical Engineering, vol. 39, no. 3, pp. 1006-1015, 2013.

[13] S. Farwa, T. Shah, N. Muhammad, N. Bibi, A. Jahangir, and S. Arshad, "An image encryption technique based on chaotic Sbox and Arnold transform," International Journal of Advanced Computer Science \& Applications, vol. 8, no. 6, pp. 360-364, 2017.

[14] F. Özkaynak, V. Çelik, and A. B. Özer, "A new S-box construction method based on the fractional-order chaotic Chen system," Signal, Image and Video Processing, vol. 11, no. 4, pp. 659-664, 2017.

[15] Y. Tian and Z. Lu, "Novel permutation-diffusion image encryption algorithm with chaotic dynamic S-box and DNA sequence operation," AIP Advances, vol. 7, no. 8, Article ID 085008, 2017.

[16] X.-M. Zhang, Y. Zheng, and H. Imai, "Relating differential distribution tables to other properties of substitution boxes," Designs, Codes and Cryptography. An International Journal, vol. 19, no. 1, pp. 45-63, 2000.

[17] X. Y. Shi, X. You, and K. Y. Lam, "A method for obtaining cryptographically strong $8 \times 8$ S-boxes," Int Conf Infor Network Appl, vol. 2, no. 3, pp. 14-20, 2002.

[18] M.-T. Tran, D.-K. Bui, and A.-D. Duong, "Gray S-box for advanced encryption standard," International Conference on Computational Intelligence and Security, pp. 253-258, 2008.

[19] M. A. Gondal, Abdul Raheem, and I. Hussain, "A Scheme for Obtaining Secure S-Boxes Based on Chaotic Baker's Map," 3D Research, vol. 5, no. 3, 2014.

[20] M. Khan, T. Shah, H. Mahmood, and M. A. Gondal, "An efficient method for the construction of block cipher with multichaotic systems," Nonlinear Dynamics, vol. 71, no. 3, pp. 489492, 2013.

[21] A. U. Rehman, J. S. Khan, J. Ahmad, and S. O. Hwang, "A New Image Encryption Scheme Based on Dynamic S-Boxes and Chaotic Maps," 3D Research, vol. 7, no. 1, article no. 7, pp. 1-8, 2016.

[22] K. Nyberg, Perfect nonlinear S-boxes, Advances in Cryptology - EUROCRYPT'91, vol. 547 of Lecture Notes in Comput. Sci., Springer, Berlin, Germany, 1991.

[23] A. F. Webster and S. E. Tavares, "On the design of S-boxes, Advances in Cryptology," in Proceedings of CRYPTO'85, Springer-Verlag, pp. 523-534, Berlin, Germany, 1986. 


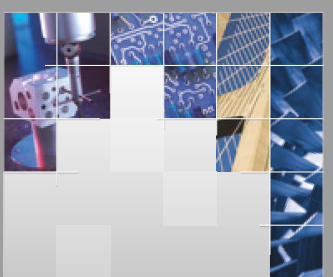

\section{Enfincering}
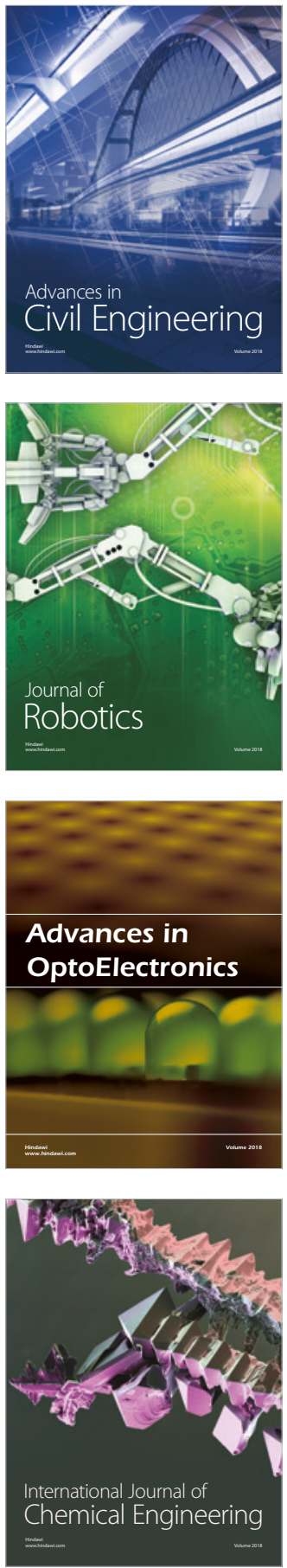

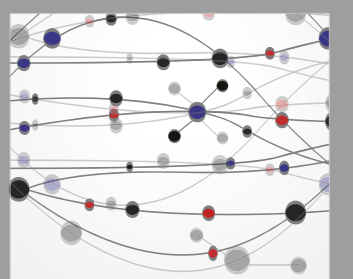

\section{Rotating \\ Machinery}

The Scientific World Journal

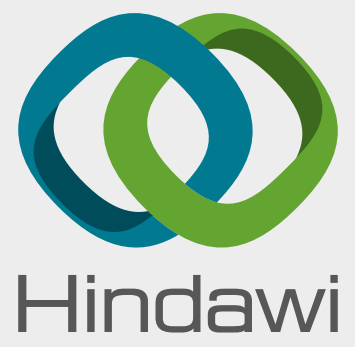

Submit your manuscripts at

www.hindawi.com
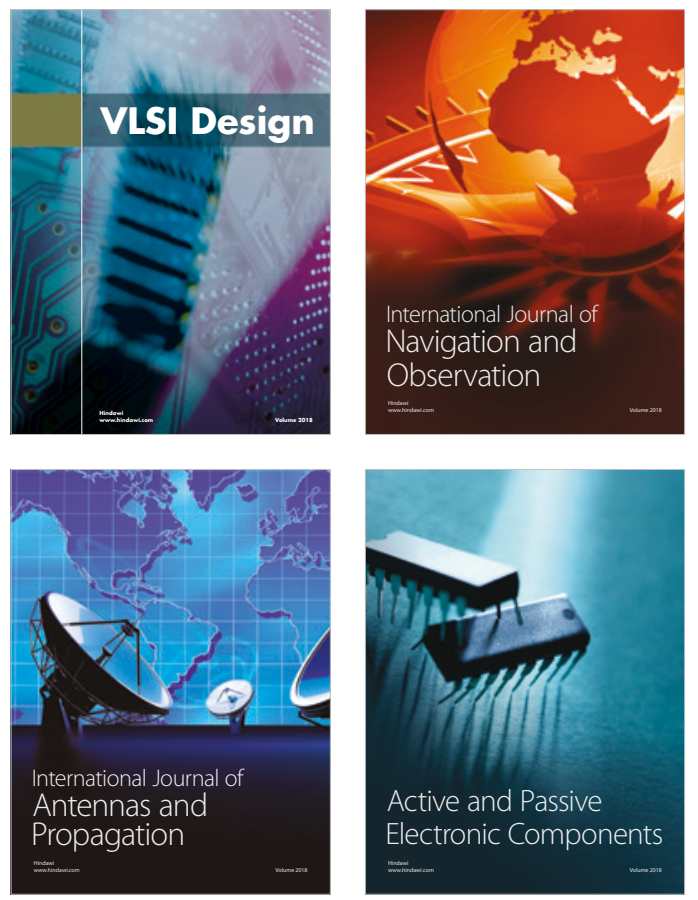
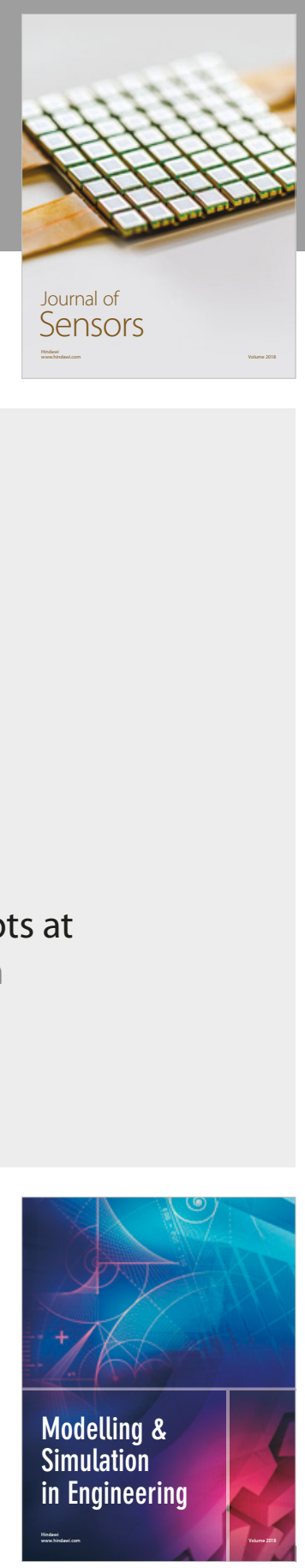

\section{Advances \\ Multimedia}
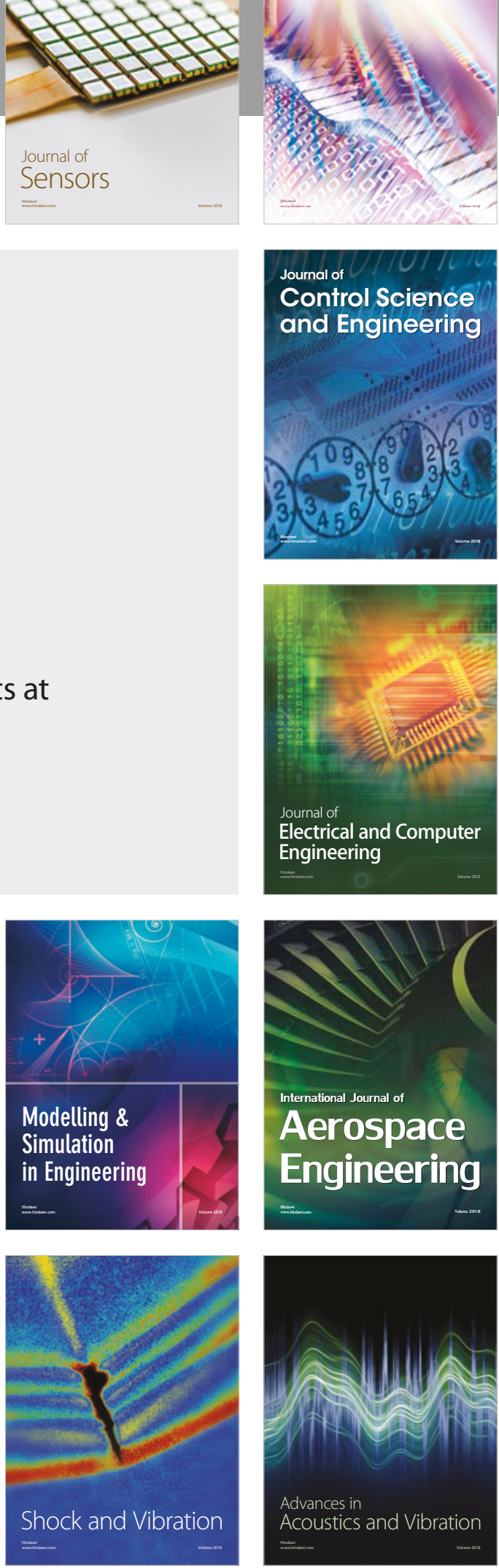